\title{
Properties and Bounds of Jensen-Type Functionals via Harmonic Convex Functions
}

\author{
Aqeel Ahmad Mughal, ${ }^{1}$ Hassan Almusawa $\mathbb{D}^{2},{ }^{2}$ Absar U1 Haq, ${ }^{3}$ and Imran Abbas Baloch $\mathbb{D}^{4,5}$ \\ ${ }^{1}$ Department of Mathematics and Statistics, University of Lahore, Lahore, Pakistan \\ ${ }^{2}$ Department of Mathematics, College of Sciences, Jazan University, Jazan 45142, Saudi Arabia \\ ${ }^{3}$ Department of Natural Sciences and Humanities, University of Engineering and Technology (Narowal Campus), \\ Lahore 54000, Pakistan \\ ${ }^{4}$ Abdus Salam School of Mathematical Sciences, GC University, Lahore, Pakistan \\ ${ }^{5}$ Higher Education Department, Government Graduate College for Boys Gulberg, Lahore, Punjab, Pakistan
}

Correspondence should be addressed to Imran Abbas Baloch; iabbasbaloch@gmail.com

Received 20 February 2021; Accepted 23 July 2021; Published 9 August 2021

Academic Editor: Ahmet Ocak Akdemir

Copyright (C) 2021 Aqeel Ahmad Mughal et al. This is an open access article distributed under the Creative Commons Attribution License, which permits unrestricted use, distribution, and reproduction in any medium, provided the original work is properly cited.

\begin{abstract}
Dragomir introduced the Jensen-type inequality for harmonic convex functions (HCF) and Baloch et al. studied its different variants, such as Jensen-type inequality for harmonic $h$-convex functions. In this paper, we aim to establish the functional form of inequalities presented by Baloch et al. and prove the superadditivity and monotonicity properties of these functionals. Furthermore, we derive the bound for these functionals under certain conditions. Furthermore, we define more generalized functionals involving monotonic nondecreasing concave function as well as evince superadditivity and monotonicity properties of these generalized functionals.
\end{abstract}

\section{Introduction}

Convexity is natural and simple notion which has found applications in business, industry, and medicine. During the study of convexity, many researchers have been fascinated by generalization of this class and have tried to find out those classes of functions which have close relation with this class (but not convex in general). Harmonic convex functions (HCFs) [1], harmonic $(\alpha, m)$-convex functions [2], harmonic $(s, m)$-convex functions $[3,4]$, and harmonic $(p,(s, m))$-convex functions [5] are among these classes. For a quick glance on importance of these classes and applications, see [6-9] and references therein. The class of harmonic convex functions (HCFs) and its different variants are very important classes that gained prominence in the theory of inequalities and applications as well as in other branches of mathematics. Many researchers have been working on the class of harmonic convex functions (HCFs) due to its significance and have been trying to explore about it more and more. During this study, recently different generalizations of the class of harmonic convex functions (HCFs) have been found, for example, see [10-13] and references therein.

The importance of the class of HCFs continuously encourages us and many other researchers to explore more about it, and the following paper is a link to it. For the better understanding of the results of present paper, we first recall some basic definitions.

Definition 1. Consider $I \subset \mathbb{R} \backslash\{0\}$. A function $f: I \longrightarrow \mathbb{R}$ is HCF on $I$ if

$$
f\left(\frac{w_{1} w_{2}}{t w_{1}+(1-t) w_{2}}\right) \leq t f\left(w_{2}\right)+(1-t) f\left(w_{1}\right)
$$

holds, for all $w_{1}, w_{2} \in I$ and $t \in[0,1]$. If we reverse the above inequality, the function $f$ becomes harmonic concave.

Remark 1 (see [14]). 
(i) The function $f(w)=\ln w$ is a HCF on the interval $(0, \infty)$, but it is not a convex function.

(ii) The function

$$
f(w)= \begin{cases}\frac{1-w}{w}, & 0<w \leq 1 \\ 0, & 1<w \leq 2 \\ \frac{w-2}{w}, & w>2\end{cases}
$$

is another example of $\mathrm{HCF}$, which is neither convex nor concave.

Baloch et al., in [7], observed some remarkable facts for the class of HCFs.

Proposition 1. For $I \subseteq \mathbb{R} \backslash\{0\}$, a function $f: I \longrightarrow \mathbb{R}$, we have the following facts:

(1) If $I \subset(0, \infty)$ and $f$ is nondecreasing and convex function, then $f$ is $\mathrm{HCF}$

(2) If $I \subset(0, \infty)$ and $f$ is nonincreasing function and harmonically convex, then $f$ is convex function

(3) If $I \subset(-\infty, 0)$ and $f$ is nondecreasing function and harmonically convex, then $f$ is convex function

(4) If $I \subset(-\infty, 0)$ and $f$ is nonincreasing function and convex, then $f$ is HCF

Varošanec, in [15], proposed the concept of h-convexity (also, see [16-18]) to unify numerous generalized aspects of convex functions. In a similar fashion, harmonic h-convexity unifies the various types of harmonic convexities.

Definition 2 (see [19]). Consider a nonnegative function $h:[0,1] \longrightarrow \mathbb{R}^{+}$. Then, the function $f: I \subseteq \mathbb{R} \backslash\{0\} \longrightarrow(0, \infty)$ is said to be harmonic $h$-convex on $I$ if the inequality

$$
f\left(\frac{w_{1} w_{2}}{t w_{1}+(1-t) w_{2}}\right) \leq h(t) f\left(w_{2}\right)+h(1-t) f\left(w_{1}\right),
$$

holds, for all $w_{1}, w_{2} \in I$ and $t \in[0,1]$. Furthermore, if we reverse inequality (3), then $f$ becomes harmonic $h$-concave.

Remark 2. We provide few examples of harmonic $h$-convex (concave) functions as follows:

(i) Obviously, with $h(t)=t$, the class of nonnegative harmonic convex (concave) functions on $I$ become a particular case of the class of harmonic $h$-convex (concave) functions on $I$.

(ii) Let $t \in(0,1)$ and $h(t)=t^{2}$. Consider a function $f:[-1,0) \cup t(0,1] \longrightarrow \mathbb{R}$ defined by $f(x)=1$, which is neither nonincreasing nor nondecreasing $h$-convex function. Therefore, $f$ is a harmonic $h$-convex function by Proposition 1 given in [7].

(iii) Let $t \in(0,1)$ and $h:(0,1) \longrightarrow(0, \infty)$ be a realvalued function such that $h(t) \geq t$ on $(0,1)$. Then, the following four functions, $h_{1}(t)=t, h_{2}(t)=t^{s}(s \in$ $(0,1)), h_{3}(t)=1 / t$, and $h_{4}(t)=1$, satisfy the conditions of the function $h$ mentioned above. Therefore, $f$ is a harmonic $h_{\alpha}$-convex function for $\alpha=1,2,3,4$ if $f: I \subseteq(0, \infty) \longrightarrow(0, \infty)$ is a nondecreasing convex function, or harmonic $s$-convex function, or harmonic Godunova-Levin function or harmonic $P$-function.

(iv) Let $f:(0, \infty) \longrightarrow(0, \infty)$ be a nondecreasing continuous function and $h:[0,1] \longrightarrow(0, \infty)$ be a continuous self-concave function such that $f\left(t w_{1}+(1-t) w_{2}\right) \leq h(t) f\left(w_{1}\right)+(1-t) f\left(w_{2}\right)$, for some $t \in(0,1)$ and all $w_{1}, w_{2} \in(0, \infty)$. Then, $f$ is a $h$-convex function by Lemma 1 of [20], and hence, $f$ is a harmonic $h$-convex function by Proposition 1 of [7].

Definition 3. A function $h: I \subseteq \mathbb{R} \longrightarrow \mathbb{R}$ is said to be a submultiplicative function if

$$
h\left(w_{1} w_{2}\right) \leq h\left(w_{1}\right) h\left(w_{2}\right)
$$

for all $w_{1}, w_{2} \in I$. If inequality (4) is reversed, then $h$ is said to be supermultiplicative function. If just equality holds in relation (4), then $h$ is said to be multiplicative function.

Definition 4. A function $h: I \subseteq \mathbb{R} \longrightarrow \mathbb{R}$ is said to be a subadditive function if

$$
h\left(w_{1}+w_{2}\right) \leq h\left(w_{1}\right)+h\left(w_{2}\right),
$$

for all $w_{1}, w_{2} \in I$. If inequality (5) is reversed, then $h$ is said to be superadditive function. If just the equality holds in relation (5), then $h$ is said to be additive function.

Jensen-type inequality for HCFs is proposed by Dragomir [21].

Theorem 1. Let $I \subseteq(0, \infty)$ be an interval. If $f: I \longrightarrow \mathbb{R}$ is HCF, then

$$
f\left(\frac{1}{\left(1 / B_{n}\right) \sum_{\alpha=1}^{n} b_{\alpha} / w_{\alpha}}\right) \leq\left(\frac{1}{B_{n}}\right) \sum_{\alpha=1}^{n} b_{\alpha} f\left(w_{\alpha}\right),
$$

Holds, for all $w_{1}, \ldots, w_{n} \in I$ and $b_{1}, \ldots, b_{n} \geq 0$ with $\sum_{\alpha=1}^{n} b_{\alpha}=B_{n}$.

In [22], Baloch et al. derived the following results:

Theorem 2. Let $I \subseteq \mathbb{R} \backslash\{0\}$. If $f: I \longrightarrow \mathbb{R}$ is HCF, then, for any finite positive sequence $\left(w_{\alpha}\right)_{\alpha=1}^{n} \in I$ and $b_{1}, \ldots, b_{n} \geq 0$ with $\sum_{\alpha=1}^{n} b_{\alpha}=B_{n}$, we have

$$
f\left(\frac{1}{\left(1 / w_{1}\right)+\left(1 / w_{n}\right)-\left(1 / B_{n}\right) \sum_{\alpha=1}^{n} b_{\alpha} / w_{\alpha}}\right) \leq f\left(w_{1}\right)+f\left(w_{n}\right)-\left(1 / B_{n}\right) \sum_{\alpha=1}^{n} b_{\alpha} f\left(w_{\alpha}\right) .
$$


Theorem 3. Let $b_{1}, \ldots, b_{n}$ be positive real numbers $(n \geq 2)$ and $B_{n}=\sum_{\alpha=1}^{n} b_{\alpha}$. If $h: I \supseteq(0,1) \longrightarrow \mathbb{R}$ is a nonnegative supermultiplicative function and if $f$ is harmonic $h$-convex function, $\left(w_{\alpha}\right)_{\alpha=1}^{n} \in I$, then

$$
f\left(\frac{1}{\left(1 / B_{n}\right) \sum_{\alpha=1}^{n} b_{\alpha} / w_{\alpha}}\right) \leq \sum_{\alpha=1}^{n} h\left(\frac{b_{\alpha}}{B_{n}}\right) f\left(w_{\alpha}\right) .
$$

Theorem 4. Let $h: I \supseteq(0,1) \longrightarrow \mathbb{R}$ be a nonnegative supermutiplicative function on $I$. Let $b_{1}, \ldots, b_{n}$ be positive real numbers $(n \geq 2)$ such that $B_{n}=\sum_{\alpha=1}^{n} b_{\alpha}$ and $\sum_{\alpha=1}^{n} h\left(b_{\alpha} / B_{n}\right) \leq 1$. If $f$ is harmonic $h$-convex on $I \subseteq \mathbb{R} \backslash\{0\}$, then, for any finite positive increasing sequence $\left(w_{\alpha}\right)_{k=1}^{n} \in I$, we have

$$
f\left(\frac{1}{\left(1 / w_{1}\right)+\left(1 / w_{n}\right)-\left(1 / B_{n}\right) \sum_{\alpha=1}^{n} b_{\alpha} / w_{\alpha}}\right) \leq f\left(w_{1}\right)+f\left(w_{n}\right)-\sum_{\alpha=1}^{n} h\left(\frac{b_{\alpha}}{B_{n}}\right) f\left(w_{\alpha}\right)
$$

If $h$ is a submultiplicative function, $\sum_{\alpha=1}^{n} h\left(b_{\alpha} / B_{n}\right) \geq 1$ and $f$ is harmonic $h$-concave then inequality (9) is reversed.

Remark 3. Importance of the class of HCFs can be guessed by the following applications in the field of mathematics:

(i) Harmonic convexity provides a useful analytic tool to calculate several known definite integrals such as $\int_{a}^{b}\left(e^{w} / x^{n}\right) \mathrm{d} w, \int_{a}^{b} e^{w^{2}} \mathrm{~d} w, \int_{a}^{b}\left(\sin w / w^{n}\right) \mathrm{d} w$, and $\int_{a}^{b}$ $\left(\cos w / w^{n}\right) \mathrm{d} x \forall n \in \mathbb{N}$, where $a, b \in(0, \infty)$, see [7]

(ii) Inequality (6) provides a very short proof of the discrete form of Hölder's inequality (see [22]) (iii) By inequalities (6) and (7), we can easily prove weighted HGA inequality (see [14])

Many researchers considered the functionals related to Jensen's inequality and tried to find properties and bound for these functionals (for example, see [23-30]). In the sequel, the set of all nonnegative $n$-tuples $\mathbf{b}=\left(b_{1}, \ldots, b_{n}\right)$, such that $B_{n}:=\sum_{\alpha=1}^{n} b_{\alpha}>0$, will be denoted with $\mathscr{B}_{n}^{0}$.

The difference between the right-hand and the left-hand side of inequalities (6)-(9) defines the following functionals:

$$
\begin{aligned}
& \mathscr{M}_{1}(f, \mathbf{w}, \mathbf{b}):=\sum_{\alpha=1}^{n} b_{\alpha} f\left(w_{\alpha}\right)-B_{n} f\left(\frac{1}{\left(1 / B_{n}\right) \sum_{\alpha=1}^{n} b_{\alpha} / w_{\alpha}}\right), \\
& \mathscr{M}_{2}(f, \mathbf{w}, \mathbf{b}):=B_{n}\left[f\left(w_{1}\right)+f\left(w_{n}\right)\right]-\sum_{\alpha=1}^{n} b_{\alpha} f\left(w_{\alpha}\right)-B_{n} f\left(\frac{1}{\left(1 / w_{1}\right)+\left(1 / w_{n}\right)-\left(1 / B_{n}\right) \sum_{\alpha=1}^{n} b_{\alpha} / w_{\alpha}}\right), \\
& \mathscr{M}_{3}(f, \mathbf{w}, \mathbf{b}):=\sum_{\alpha=1}^{n} h\left(\frac{b_{\alpha}}{B_{n}}\right) f\left(w_{\alpha}\right)-f\left(\frac{1}{\left(1 / B_{n}\right) \sum_{\alpha=1}^{n} b_{\alpha} / w_{\alpha}}\right), \\
& \mathscr{M}_{4}(f, \mathbf{w}, \mathbf{b}):=f\left(w_{1}\right)+f\left(w_{n}\right)-\sum_{\alpha=1}^{n} h\left(\frac{b_{\alpha}}{B_{n}}\right) f\left(w_{\alpha}\right)-f\left(\frac{1}{\left(1 / w_{1}\right)+\left(1 / w_{n}\right)-\left(1 / B_{n}\right) \sum_{\alpha=1}^{n} b_{\alpha} / w_{\alpha}}\right) .
\end{aligned}
$$

For a fixed function $f$ and $n$-tuple $\mathbf{w}, \mathscr{M}_{1}(f, \mathbf{w},$.$) ,$ $\mathscr{M}_{2}(f, \mathbf{w},),. \mathscr{M}_{3}(f, \mathbf{w},$.$) , and \mathscr{M}_{4}(f, \mathbf{w},$.$) can be considered$ as functions on $\mathscr{B}_{n}^{0}$, which is a convex subset in $\mathbb{R}^{n}$. Furthermore, because of inequalities (6)-(9), we have $\mathscr{M}_{1}(f, \mathbf{w}$, b) $\geq 0, \mathscr{M}_{2}(f, \mathbf{w}, \mathbf{b}) \geq 0, \mathscr{M}_{3}(f, \mathbf{w}, \mathbf{b}) \geq 0$, and $\mathscr{M}_{4}(f, \mathbf{w}, \mathbf{b}) \geq$ 0 , for all $\mathbf{b} \in \mathscr{B}_{n}^{0}$.

\section{Main Results}

In this section, we establish some properties of functionals related to Jensen-type inequalities for HCFs.
Theorem 5. Let $\mathbf{b}=\left(b_{1}, \ldots, b_{n}\right)$ and $\mathbf{c}=\left(c_{1}, \ldots, c_{n}\right)$ be two $n$-tuples from $\mathscr{B}_{n}^{0}$. Let $I \subseteq(0, \infty)$ be an interval. If $f: I \longrightarrow \mathbb{R}$ is a HCF, $h: I \supseteq(0,1) \longrightarrow \mathbb{R}$ is a nonnegative multiplicative and additive function on $J$, and if $\mathbf{w}=\left(w_{1}, \ldots, w_{n}\right) \in I^{n}$, $h(\alpha)+h(1-\alpha) \geq 2$, then $\mathscr{M}_{i}(f, \mathbf{w},$.$) , for i=1,2,3,4$, defined by (10)-(12) are superadditive on $\mathscr{B}_{n}^{0}$, i.e.,

$$
\mathscr{M}_{i}(f, \mathbf{w}, \mathbf{b}+\mathbf{c}) \geq \mathscr{M}_{i}(f, \mathbf{w}, \mathbf{b})+\mathscr{M}_{i}(f, \mathbf{w}, \mathbf{c}) \geq 0,
$$

for $i=1,2,3,4$.

Proof. Take $i=1$ in (28) and starting from definition, we have 


$$
\begin{aligned}
\mathscr{M}_{1}(f, \mathbf{w}, \mathbf{b}+\mathbf{c}) & =\sum_{\alpha=1}^{n}\left(b_{\alpha}+c_{\alpha}\right) f\left(w_{\alpha}\right)-\left(B_{n}+C_{n}\right) f\left(\frac{1}{\left(1 / B_{n}+C_{n}\right) \sum_{\alpha=1}^{n} b_{\alpha}+c_{\alpha} / w_{\alpha}}\right) \\
& =\sum_{\alpha=1}^{n} b_{\alpha} f\left(w_{\alpha}\right)+\sum_{\alpha=1}^{n} c_{\alpha} f\left(w_{\alpha}\right)-\left(B_{n}+C_{n}\right) f\left(\frac{1}{\left(1 / B_{n}+C_{n}\right) \sum_{\alpha=1}^{n} b_{\alpha}+c_{\alpha} / w_{\alpha}}\right),
\end{aligned}
$$

while, after arranging and harmonic convexity of $f$, yields

$$
\begin{aligned}
f\left(\frac{1}{\left(1 / B_{n}+C_{n}\right) \sum_{\alpha=1}^{n} b_{\alpha}+c_{\alpha} / w_{\alpha}}\right) & =f\left(\frac{1}{\left(B_{n} / B_{n}+C_{n}\right) \cdot\left(1 / B_{n}\right) \sum_{\alpha=1}^{n} b_{\alpha} / w_{\alpha}+\left(C_{n} / B_{n}+C_{n}\right) \cdot\left(1 / C_{n}\right) \sum_{\alpha=1}^{n} c_{\alpha} / w_{\alpha}}\right) \\
& \leq \frac{B_{n}}{B_{n}+C_{n}} f\left(\frac{1}{\left(1 / B_{n}\right) \sum_{\alpha=1}^{n} b_{\alpha} / w_{\alpha}}\right)+\frac{C_{n}}{B_{n}+C_{n}} f\left(\frac{1}{\left(1 / C_{n}\right) \sum_{\alpha=1}^{n} c_{\alpha} / w_{\alpha}}\right) .
\end{aligned}
$$
obtain

Finally, combining relation (15) and inequality (16), we

$$
\begin{gathered}
\mathscr{M}_{1}(f, \mathbf{w}, \mathbf{b}+\mathbf{c}) \geq \sum_{\alpha=1}^{n} b_{\alpha} f\left(w_{\alpha}\right)+\sum_{\alpha=1}^{n} c_{\alpha} f\left(w_{\alpha}\right)-B_{n} f\left(\frac{1}{\left(1 / B_{n}\right) \sum_{\alpha=1}^{n} b_{\alpha} / w_{\alpha}}\right) \\
-C_{n} f\left(\frac{1}{\left(1 / C_{n}\right) \sum_{\alpha=1}^{n} c_{\alpha} / w_{\alpha}}\right)=\mathscr{M}_{1}(f, \mathbf{w}, \mathbf{b})+\mathscr{M}_{1}(f, \mathbf{w}, \mathbf{c}) .
\end{gathered}
$$

Now, taking $i=2$ in (28) and starting from the definition, we have

$$
\begin{aligned}
\mathscr{M}_{2}(f, \mathbf{w}, \mathbf{b}+\mathbf{c})= & \left(B_{n}+C_{n}\right)\left[f\left(w_{1}\right)+f\left(w_{n}\right)\right]-\sum_{\alpha=1}^{n}\left(b_{\alpha}+c_{\alpha}\right) f\left(w_{\alpha}\right) \\
& -\left(B_{n}+C_{n}\right) f\left(\frac{1}{\left(1 / w_{1}\right)+\left(1 / w_{n}\right)-\left(1 / B_{n}+C_{n}\right) \sum_{\alpha=1}^{n} b_{\alpha}+c_{\alpha} / w_{\alpha}}\right) \\
= & B_{n}\left[f\left(w_{1}\right)+f\left(w_{n}\right)\right]+C_{n}\left[f\left(w_{1}\right)+f\left(w_{n}\right)\right] \\
& -\sum_{\alpha=1}^{n} b_{\alpha} f\left(w_{\alpha}\right)-\sum_{\alpha=1}^{n} c_{\alpha} f\left(w_{\alpha}\right)-\left(B_{n}+C_{n}\right) \\
& f\left(\frac{1}{\left(1 / w_{1}\right)+\left(1 / w_{n}\right)-\left(1 / B_{n}+C_{n}\right) \sum_{\alpha=1}^{n} b_{\alpha}+c_{\alpha} / w_{\alpha}}\right),
\end{aligned}
$$

while, after arranging and harmonic convexity of $f$, yields 


$$
\begin{aligned}
& f\left(\frac{1}{\left(1 / w_{1}\right)+\left(1 / w_{n}\right)-\left(1 / B_{n}+C_{n}\right) \sum_{\alpha=1}^{n} b_{\alpha}+c_{\alpha} / w_{\alpha}}\right)=f\left(\frac{1}{\left(1 / B_{n}+C_{n}\right) \sum_{\alpha=1}^{n}\left(b_{\alpha}+c_{\alpha}\right)\left(\left(1 / w_{1}\right)+\left(1 / w_{n}\right)-\left(1 / w_{\alpha}\right)\right)}\right) \\
& =f\left(\frac{1}{\left(B_{n} / B_{n}+C_{n}\right) \sum_{\alpha=1}^{n}\left(b_{\alpha} / B_{n}\right)\left(\left(1 / w_{1}\right)+\left(1 / w_{n}\right)-\left(1 / w_{\alpha}\right)\right)+\left(C_{n} / B_{n}+C_{n}\right) \sum_{\alpha=1}^{n}\left(c_{\alpha} / C_{n}\right)\left(\left(1 / w_{1}\right)+\left(1 / w_{n}\right)-\left(1 / w_{\alpha}\right)\right)}\right) \\
& \leq \frac{B_{n}}{B_{n}+C_{n}} f\left(\frac{1}{\sum_{\alpha=1}^{n}\left(b_{\alpha} / B_{n}\right)\left(\left(1 / w_{1}\right)+\left(1 / w_{n}\right)-\left(1 / w_{\alpha}\right)\right)}\right)+\frac{C_{n}}{B_{n}+C_{n}} f\left(\frac{1}{\sum_{\alpha=1}^{n}\left(c_{\alpha} / C_{n}\right)\left(\left(1 / w_{1}\right)+\left(1 / w_{n}\right)-\left(1 / w_{\alpha}\right)\right)}\right) \\
& =\frac{B_{n}}{B_{n}+C_{n}} f\left(\frac{1}{\left(1 / w_{1}\right)+\left(1 / w_{n}\right)-\left(1 / B_{n}\right) \sum_{\alpha=1}^{n} b_{\alpha} / w_{\alpha}}\right)+\frac{C_{n}}{B_{n}+C_{n}} f\left(\frac{1}{\left(1 / w_{1}\right)+\left(1 / w_{n}\right)-\left(1 / C_{n}\right) \sum_{\alpha=1}^{n} c_{\alpha} / w_{\alpha}}\right) .
\end{aligned}
$$

Finally, combining relation (18) and inequality (19), we obtain

$$
\begin{aligned}
& \mathscr{M}_{2}(f, \mathbf{w}, \mathbf{b}+\mathbf{c}) \geq B_{n}\left[f\left(w_{1}\right)+f\left(w_{n}\right)\right]+C_{n}\left[f\left(w_{1}\right)+f\left(w_{n}\right)\right]-\sum_{\alpha=1}^{n} b_{\alpha} f\left(w_{\alpha}\right) \\
& -\sum_{\alpha=1}^{n} c_{\alpha} f\left(w_{\alpha}\right)-\left(B_{n}+C_{n}\right) \cdot \frac{B_{n}}{B_{n}+C_{n}} f\left(\frac{1}{\left(1 / w_{1}\right)+\left(1 / w_{n}\right)-\left(1 / B_{n}\right) \sum_{\alpha=1}^{n} b_{\alpha} / w_{\alpha}}\right) \\
& -\left(B_{n}+C_{n}\right) \cdot \frac{C_{n}}{B_{n}+C_{n}} f\left(\frac{1}{\left(1 / w_{1}\right)+\left(1 / w_{n}\right)-\left(1 / C_{n}\right) \sum_{\alpha=1}^{n} c_{\alpha} / w_{\alpha}}\right)=\mathscr{M}_{2}(f, \mathbf{w}, \mathbf{b})+\mathscr{M}_{2}(f, \mathbf{w}, \mathbf{c}) .
\end{aligned}
$$
have

Taking $i=3$ in (28) and starting from the definition, we

$$
\begin{aligned}
\mathscr{M}_{3}(f, \mathbf{w}, \mathbf{b}+\mathbf{c})= & \sum_{\alpha=1}^{n} h\left(\frac{b_{\alpha}+c_{\alpha}}{B_{n}+C_{n}}\right) f\left(w_{\alpha}\right)-f\left(\frac{1}{\left(1 / B_{n}+C_{n}\right) \sum_{\alpha=1}^{n} b_{\alpha}+c_{\alpha} / w_{\alpha}}\right) \\
= & \sum_{\alpha=1}^{n} h\left(\frac{b_{\alpha}}{B_{n}+C_{n}}\right) f\left(w_{\alpha}\right)+\sum_{\alpha=1}^{n} h\left(\frac{c_{\alpha}}{B_{n}+C_{n}}\right) f\left(w_{\alpha}\right) \\
& -f\left(\frac{1}{\left(1 / B_{n}+C_{n}\right) \sum_{\alpha=1}^{n} b_{\alpha}+c_{\alpha} / w_{\alpha}}\right) \\
= & \sum_{\alpha=1}^{n} h\left(\frac{B_{n}}{B_{n}+C_{n}}\right) h\left(\frac{b_{\alpha}}{B_{n}}\right) f\left(w_{\alpha}\right)+\sum_{\alpha=1}^{n} h\left(\frac{C_{n}}{B_{n}+C_{n}}\right) h\left(\frac{c_{\alpha}}{C_{n}}\right) f\left(w_{\alpha}\right) \\
& -f\left(\frac{1}{\left(1 / B_{n}+C_{n}\right) \sum_{\alpha=1}^{n} b_{\alpha}+c_{\alpha} / w_{\alpha}}\right)
\end{aligned}
$$

while, after arranging and harmonic $h$-convexity of $f$, yields 


$$
\begin{aligned}
f\left(\frac{1}{\left(1 / B_{n}+C_{n}\right) \sum_{\alpha=1}^{n}\left(p_{i}+c_{\alpha} / w_{\alpha}\right)}\right) & =f\left(\frac{1}{\left(B_{n} / B_{n}+C_{n}\right) \cdot\left(1 / B_{n}\right) \sum_{\alpha=1}^{n}\left(b_{\alpha} / w_{\alpha}\right)+\left(C_{n} / B_{n}+C_{n}\right) \cdot\left(1 / C_{n}\right) \sum_{\alpha=1}^{n} c_{\alpha} / w_{\alpha}}\right) \\
& \leq h\left(\frac{B_{n}}{B_{n}+C_{n}}\right) f\left(\frac{1}{\left(1 / B_{n}\right) \sum_{\alpha=1}^{n} b_{\alpha} / w_{\alpha}}\right)+h\left(\frac{C_{n}}{B_{n}+C_{n}}\right) f\left(\frac{1}{\left(1 / C_{n}\right) \sum_{\alpha=1}^{n} c_{\alpha} / w_{\alpha}}\right) \\
& =h\left(\frac{B_{n}}{B_{n}+C_{n}}\right) f\left(\frac{1}{\left(1 / B_{n}\right) \sum_{\alpha=1}^{n} b_{\alpha} / w_{\alpha}}\right)+h\left(\frac{C_{n}}{B_{n}+C_{n}}\right) f\left(\frac{1}{\left(1 / C_{n}\right) \sum_{\alpha=1}^{n} c_{\alpha} / w_{\alpha}}\right)
\end{aligned}
$$

Finally, combining relation (21) and inequality (22), we obtain

$$
\begin{aligned}
\mathscr{M}_{3}(f, \mathbf{w}, \mathbf{b}+\mathbf{c}) \geq & h\left(\frac{B_{n}}{B_{n}+C_{n}}\right) \sum_{\alpha=1}^{n} h\left(\frac{b_{\alpha}}{B_{n}}\right) f\left(w_{\alpha}\right)+h\left(\frac{C_{n}}{B_{n}+C_{n}}\right) \sum_{\alpha=1}^{n} h\left(\frac{c_{\alpha}}{C_{n}}\right) f\left(w_{\alpha}\right) \\
& -h\left(\frac{B_{n}}{B_{n}+C_{n}}\right) f\left(\frac{1}{\left(1 / B_{n}\right) \sum_{\alpha=1}^{n} b_{\alpha} / w_{\alpha}}\right)-h\left(\frac{C_{n}}{B_{n}+C_{n}}\right) f\left(\frac{1}{\left(1 / C_{n}\right) \sum_{\alpha=1}^{n} c_{\alpha} / w_{\alpha}}\right) \\
= & h\left(\frac{B_{n}}{B_{n}+C_{n}}\right)\left[\sum_{\alpha=1}^{n} h\left(\frac{b_{\alpha}}{B_{n}}\right) f\left(w_{\alpha}\right)-f\left(\frac{1}{\left(1 / B_{n}\right) \sum_{\alpha=1}^{n} b_{\alpha} / w_{\alpha}}\right)\right] \\
& +h\left(\frac{C_{n}}{B_{n}+C_{n}}\right)\left[\sum_{\alpha=1}^{n} h\left(\frac{c_{\alpha}}{C_{n}}\right) f\left(w_{\alpha}\right)-f\left(\frac{1}{\left(1 / C_{n}\right) \sum_{i=1}^{n} c_{\alpha} / w_{\alpha}}\right)\right] \\
\geq & {\left[\sum_{\alpha=1}^{n} h\left(\frac{b_{\alpha}}{B_{n}}\right) f\left(w_{\alpha}\right)-f\left(\frac{1}{\left(1 / B_{n}\right) \sum_{\alpha=1}^{n}\left(b_{\alpha} / w_{\alpha}\right)}\right)\right]+\left[\sum_{\alpha=1}^{n} h\left(\frac{c_{\alpha}}{C_{n}}\right) f\left(w_{\alpha}\right)-f\left(\frac{1}{\left(1 / C_{n}\right) \sum_{\alpha=1}^{n} c_{\alpha} / w_{\alpha}}\right)\right] } \\
= & \mathscr{M}_{3}(f, \mathbf{w}, \mathbf{b})+\mathscr{M}_{3}(f, \mathbf{w}, \mathbf{c}) .
\end{aligned}
$$

Similarly, it can be proved that

$$
\mathscr{M}_{4}(f, \mathbf{w}, \mathbf{b}+\mathbf{c}) \geq \mathscr{M}_{4}(f, \mathbf{w}, \mathbf{b})+\mathscr{M}_{4}(f, \mathbf{w}, \mathbf{c}) .
$$

Theorem 6. Let $\mathbf{b}=\left(b_{1}, \ldots, b_{n}\right)$ and $\mathbf{c}=\left(c_{1}, \ldots, c_{n}\right)$ be two $n$-tuples from $\mathscr{B}_{n}^{0}$ such that $\mathbf{b} \geq \mathbf{c}$, (i.e., $b_{\alpha} \geq c_{\alpha}, \alpha=1, \ldots, n$ ). Let $I \subseteq(0, \infty)$ be an interval. If $f: I \longrightarrow \mathbb{R}$ is a HCF and if $\mathbf{w}=\left(w_{1}, \ldots, w_{n}\right) \in I^{n}$, then $\mathscr{M}_{i}(f, \mathbf{w},$.$) , for i=1,2,3,4$, defined by (10)-(12) satisfy the following inequality:

$$
\mathscr{M}_{i}(f, \mathbf{w}, \mathbf{b}) \geq \mathscr{M}_{i}(f, \mathbf{w}, \mathbf{c}),
$$

on $\mathscr{B}_{n}^{0}$.

Proof. The monotonicity property follows directly from superadditivity. Since $\mathbf{b} \geq \mathbf{c}, \mathbf{b}$ can be represented as the sum of two $n$-tuples: $\mathbf{b}-\mathbf{c}$ and $\mathbf{c}$. Applying (28), we have

$$
\mathscr{M}_{i}(f, \mathbf{w}, \mathbf{b})=\mathscr{M}_{i}(f, \mathbf{w},(\mathbf{b}-\mathbf{c})+\mathbf{c}) \geq \mathscr{M}_{i}(f, \mathbf{w},(\mathbf{b}-\mathbf{c}))+\mathscr{M}_{i}(f, \mathbf{w}, \mathbf{c}) .
$$

Finally, $\mathscr{M}_{i}(f, \mathbf{w},(\mathbf{b}-\mathbf{c})) \geq 0$ by (10)-(12). So, we have that $\mathscr{M}_{i}(f, \mathbf{w}, \mathbf{b}) \geq \mathscr{M}_{i}(f, \mathbf{w}, \mathbf{c})$, which proves the theorem.

Theorem 7. Let $\mathbf{b}=\left(b_{1}, \ldots, b_{n}\right)$ and $\mathbf{c}=\left(c_{1}, \ldots, c_{n}\right)$ be two $n$-tuples from $\mathscr{B}_{n}^{0}$. Let $m$ and $M$ be real constants such that

$$
\begin{aligned}
& m \geq 0, \quad b_{\alpha}-m c_{\alpha} \geq 0, \\
& M c_{\alpha}-b_{\alpha} \geq 0, \quad \alpha=1, \ldots n .
\end{aligned}
$$

Let $I \subseteq(0, \infty)$ be an interval. If $f: I \longrightarrow \mathbb{R}$ is a HCF and if $\mathbf{w}=\left(w_{1}, \ldots, w_{n}\right) \in I^{n}$ be any $n$-tuple, then

$$
M \mathscr{M}_{i}(f, \mathbf{w}, \mathbf{c}) \geq \mathscr{M}_{i}(f, \mathbf{w}, \mathbf{b}) \geq m \mathscr{M}_{i}(f, \mathbf{w}, \mathbf{c}),
$$

for $i=1,2,3,4$.

Proof. Since $m \geq 0, b_{\alpha}-m c_{\alpha} \geq 0$ and $M c_{\alpha}-b_{\alpha} \geq 0, \alpha=1$, $\ldots n$, this implies that $\mathbf{b}-m \mathbf{c}$ and $M \mathbf{c}-\mathbf{b}$ are in $\mathscr{B}_{n}^{0}$. Then, by Theorem 5 , we obtain 


$$
\begin{aligned}
\mathscr{M}_{i}(f, \mathbf{w}, \mathbf{b}) & \geq \mathscr{M}_{i}(f, \mathbf{w},(\mathbf{b}-m \mathbf{c}))+\mathscr{M}_{i}(f, \mathbf{w}, m \mathbf{c}) \\
& \geq m \mathscr{M}_{i}(f, \mathbf{w}, \mathbf{c}) .
\end{aligned}
$$

Similarly, we obtain

$$
\mathscr{M}_{i}(f, \mathbf{w}, \mathbf{b}) \leq M \mathscr{M}_{i}(f, \mathbf{w}, \mathbf{c}),
$$

that is,

$$
M \mathscr{M}_{i}(f, \mathbf{w}, \mathbf{c}) \geq \mathscr{M}_{i}(f, \mathbf{w}, \mathbf{b}) \geq m \mathscr{M}_{i}(f, \mathbf{w}, \mathbf{c}) .
$$

Corollary 1. Let $\mathbf{b}, \mathbf{w}, f$, and functional $\mathscr{M}_{i}$ be as in Theorem 5. Then,

$$
\max _{1 \leq k \leq n}\left\{b_{\alpha}\right\} \mathscr{M}_{i}^{\mathcal{N}}(f, \mathbf{w}) \geq \mathscr{M}_{i}(f, \mathbf{w}, \mathbf{b}) \geq \min _{1 \leq k \leq n}\left\{b_{\alpha}\right\} \mathscr{M}_{i}^{\mathcal{N}}(f, \mathbf{w}) \quad(\text { for } i=1,2)
$$

where

$$
\begin{aligned}
& \mathscr{M}_{1}^{\mathcal{N}}(f, \mathbf{w})=\sum_{\alpha=1}^{n} f\left(w_{\alpha}\right)-n f\left(\frac{1}{(1 / n) \sum_{\alpha=1}^{n} 1 / w_{\alpha}}\right), \\
& \mathscr{M}_{2}^{\mathcal{N}}(f, \mathbf{w})=n\left[f\left(w_{1}\right)+f\left(w_{n}\right)\right]-\sum_{\alpha=1}^{n} f\left(w_{\alpha}\right)-n f\left(\frac{1}{\left(1 / w_{1}\right)+\left(1 / w_{n}\right)-(1 / n) \sum_{\alpha=1}^{n} 1 / w_{\alpha}}\right) . \\
& \mathscr{M}_{i}(f, \mathbf{w}, \mathbf{b}) \geq \mathscr{M}_{i}\left(f, \mathbf{w}, \mathbf{b}_{\text {min }}\right) .
\end{aligned}
$$

Proof. Let $\mathbf{b}_{\text {min }} \in \mathscr{B}_{n}^{0}$ be a constant $n$-tuple, i.e.,

$$
\mathbf{b}_{\min }=\left(\min _{1 \leq k \leq n}\left\{b_{\alpha}\right\}, \ldots, \min _{1 \leq k \leq n}\left\{b_{\alpha}\right\}\right) \text {. }
$$

On the contrary,

Then, for any $\mathbf{b} \in \mathscr{B}_{n}^{0}$, we have $\mathbf{b} \geq \mathbf{b}_{\min }$. So, by applying Theorem 6, we have

$$
\begin{aligned}
& \mathscr{M}_{1}\left(f, \mathbf{w}, \mathbf{b}_{\text {min }}\right)=\min _{1 \leq k \leq n}\left\{b_{\alpha}\right\}\left\{\sum_{\alpha=1}^{n} f\left(w_{\alpha}\right)-n f\left(\frac{1}{(1 / n) \sum_{\alpha=1}^{n} 1 / w_{\alpha}}\right)\right\} \\
& \mathscr{M}_{1}\left(f, \mathbf{w}, \mathbf{b}_{\text {min }}\right)=\min _{1 \leq k \leq n}\left\{b_{\alpha}\right\}\left\{n\left[f\left(w_{1}\right)+f\left(w_{n}\right)\right]-\sum_{\alpha=1}^{n} f\left(w_{\alpha}\right)-n f\left(\frac{1}{\left(1 / w_{1}\right)+\left(1 / w_{n}\right)-(1 / n) \sum_{\alpha=1}^{n} 1 / w_{\alpha}}\right)\right\}
\end{aligned}
$$

i.e., $\mathscr{M}_{1}\left(f, \mathbf{w}, \mathbf{b}_{\text {min }}\right)=\min _{1 \leq k \leq n}\left\{b_{\alpha}\right\} \mathscr{M}_{i}^{\mathcal{N}}(f, \mathbf{w})$. So, it proves the right-hand side of inequality (35). The left-hand inequality is obtained similarly by exchanging the role of min and max.

To present our next results, we need to introduce the following notations:

$$
\begin{aligned}
& J(\mathbb{R}):=\left\{\mathbf{b}=\left\{b_{\alpha}\right\}_{k \in \mathbb{N}}: b_{\alpha} \in \mathbb{R} \quad \text { are } \quad\right. \text { such that } \\
& \left.B_{K}:=\sum_{\alpha \in I} b_{\alpha} \neq 0, \text { for all } K \in P_{f}(\mathbb{N})\right\}
\end{aligned}
$$

$F^{+}(C, \mathbb{R}):=\{f \in F(C, \mathbb{R}): f(x)>0$, for all $x \in C\}$

$J^{+}(\mathbb{R}):=\left\{\mathbf{b} \in J(\mathbb{R}): b_{\alpha} \geq 0\right.$, for all $\left.k \in \mathbb{N}\right\}$

$J_{*}(\mathbb{R}):=\left\{\mathbf{w}=\left\{w_{\alpha}\right\}_{\alpha \in \mathbb{N}}: w_{\alpha} \in C\right.$, for all $\left.k \in \mathbb{N}\right\}$

$P_{f}(\mathbb{N}):=\{K \subset \mathbb{N}: K$ is finite $\}$

$H \operatorname{Conv}(C, \mathbb{R}):=$ the cone of all HCFs on $\mathrm{C}$

$F(C, \mathbb{R}):=$ the linear space of all real functions on $C$

Now, we consider more general functionals: 


$$
\begin{aligned}
& \mathscr{D}_{1}(f, K, \mathbf{b}, \mathbf{w} ; \Psi):=B_{K} \Psi\left[\frac{1}{B_{K}} \sum_{\alpha \in K} b_{\alpha} f\left(w_{\alpha}\right)-f\left(\frac{1}{\left(1 / B_{K}\right) \sum_{\alpha \in K} b_{\alpha} / w_{\alpha}}\right)\right], \\
& \mathscr{D}_{2}(f, K, \mathbf{b}, \mathbf{w} ; \Psi):=B_{K} \Psi\left[f\left(W_{1}\right)+f\left(X_{2}\right) \frac{1}{B_{K}} \sum_{\alpha \in K} b_{\alpha} f\left(w_{\alpha}\right)-f\left(\frac{1}{\left(1 / w_{1}\right)+\left(1 / x_{2}\right)-\left(1 / B_{K}\right) \sum_{\alpha \in K} b_{\alpha} / w_{\alpha}}\right)\right], \\
& \mathscr{D}_{3}(f, K, \mathbf{b}, \mathbf{w} ; \Psi):=\Psi\left[\sum_{\alpha \in K} h\left(\frac{b_{\alpha}}{B_{K}}\right) f\left(w_{\alpha}\right)-f\left(\frac{1}{\left(1 / B_{K}\right) \sum_{\alpha \in K} b_{\alpha} / w_{\alpha}}\right)\right], \\
& \mathscr{D}_{4}(f, K, \mathbf{b}, \mathbf{w} ; \Psi):=\Psi\left[f\left(W_{1}\right)+f\left(X_{2}\right) \frac{1}{B_{K}} \sum_{\alpha \in K} h\left(\frac{b_{\alpha}}{B_{K}}\right) f\left(w_{\alpha}\right)-f\left(\frac{1}{\left(1 / w_{1}\right)+\left(1 / x_{2}\right)-\left(1 / B_{K}\right) \sum_{\alpha \in K} b_{\alpha} / w_{\alpha}}\right)\right],
\end{aligned}
$$

where $f \in H \operatorname{Conv}(C, \mathbb{R}), K \in P_{f}(\mathbb{N}), \quad \mathbf{b} \in J^{+}(\mathbb{R}), \quad \mathbf{w} \in J_{*}$ $(C)$, and $\Psi:(0, \infty) \longrightarrow \mathbb{R}$ is a convex function whose properties will determine the behavior of functional $\mathscr{D}_{i}, i=1,2,3,4$, as follows. Obviously, for $\Psi(t)=t$, we recapture, from functional $\mathscr{D}_{i}$, the functional $\mathscr{M}_{i}$ considered in Theorem 5.

First of all, we observe that, by Jensen-type inequality, the functional $\mathscr{D}_{i}$ is well defined and positive homogenous in the third variable, that is,

$$
\mathscr{D}_{i}(f, K, m \mathbf{b}, \mathbf{w} ; \Psi)=m \mathscr{D}_{i}(f, K, \mathbf{b}, \mathbf{w} ; \Psi),
$$

for any $m>0$ and $\mathbf{b} \in J^{+}(\mathbb{R})$.

The following result concerning the superadditivity and the monotonicity of the functional $\mathscr{D}_{i}, i=1,2$, as function of weights holds.

Theorem 8. Let $f \in H \operatorname{Conv}(C, \mathbb{R}), K \in P_{f}(\mathbb{N}), \mathbf{w} \in J_{*}(C)$, and $\Psi:(0, \infty) \longrightarrow \mathbb{R}$ be monotonic nondecreasing and concave function. (i) If $\mathbf{b}, \mathbf{c} \in J^{+}(\mathbb{R})$, then

$\mathscr{D}_{i}(f, K, \mathbf{b}+\mathbf{c}, \mathbf{w} ; \Psi) \geq \mathscr{D}_{i}(f, K, \mathbf{b}, \mathbf{w} ; \Psi)+\mathscr{D}_{i}(f, K, \mathbf{c}, \mathbf{w} ; \Psi)$.

That is, $\mathscr{D}_{i}$ is superadditive as a function of weights.

(ii) If $\mathbf{b}, \mathbf{c} \in J^{+}(\mathbb{R})$, with $b \geq c$, meaning that $b_{i} \geq c_{i}$, for each $i \in \mathbb{N}$ and $\Psi:(0, \infty) \longrightarrow(0, \infty)$, then

$$
\mathscr{D}_{i}(f, K, \mathbf{b}, \mathbf{w} ; \Psi) \geq \mathscr{D}_{i}(f, K, \mathbf{c}, \mathbf{w} ; \Psi) \geq 0 .
$$

That is, $\mathscr{D}_{i}$ is monotonic nondecreasing as function of weights.

Proof

(i) Let $\mathbf{b}, \mathbf{c} \in J^{+}(\mathbb{R})$; then, by the harmonic convexity of $f$ on $C$,

$$
\begin{aligned}
& \frac{1}{B_{K}+C_{K}} \sum_{\alpha \in K}\left(b_{\alpha}+c_{\alpha}\right) f\left(w_{\alpha}\right)-f\left(\frac{1}{\left(1 / B_{K}+B_{K}\right) \sum_{\alpha \in K}\left(b_{\alpha}+c_{\alpha}\right) / w_{\alpha}}\right) \\
& =\frac{B_{K}\left(\left(1 / B_{K}\right) \sum_{\alpha \in K} b_{\alpha} f\left(w_{\alpha}\right)\right)+C_{K}\left(\left(1 / C_{K}\right) \sum_{\alpha \in K} c_{\alpha} f\left(w_{\alpha}\right)\right)}{B_{K}+C_{K}} \\
& \quad-f\left(\frac{1}{\left(B_{K} / B_{K}+C_{K}\right) \cdot\left(1 / B_{K}\right) \sum_{\alpha \in K} b_{\alpha} / w_{\alpha}+\left(C_{K} / B_{K}+C_{K}\right) \cdot\left(1 / C_{K}\right) \sum_{\alpha \in K} c_{\alpha} / w_{\alpha}}\right) \\
& \geq \frac{B_{K}\left(\left(1 / B_{K}\right) \sum_{\alpha \in K} b_{\alpha} f\left(w_{\alpha}\right)\right)+C_{K}\left(\left(1 / C_{K}\right) \sum_{\alpha \in K} c_{\alpha} f\left(w_{\alpha}\right)\right)}{B_{K}+C_{K}}
\end{aligned}
$$




$$
\begin{aligned}
& -\frac{B_{K} f\left(1 /\left(1 / B_{K}\right) \sum_{\alpha \in K} b_{\alpha} / w_{\alpha}\right)+C_{K} f\left(1 /\left(1 / C_{K}\right) \sum_{\alpha \in K} c_{\alpha} / w_{\alpha}\right)}{B_{K}+C_{K}} \\
= & \frac{B_{K}\left[\left(1 / B_{K}\right) \sum_{\alpha \in K} b_{\alpha} f\left(w_{\alpha}\right)-f\left(1 /\left(1 / B_{K}\right) \sum_{\alpha \in K} b_{\alpha} / w_{\alpha}\right)\right]}{B_{K}+C_{K}} \\
& +\frac{C_{K}\left[\left(1 / C_{K}\right) \sum_{\alpha \in K} c_{\alpha} f\left(w_{\alpha}\right)-f\left(1 /\left(1 / C_{K}\right) \sum_{\alpha \in K} c_{\alpha} / w_{\alpha}\right)\right]}{B_{K}+C_{K}} .
\end{aligned}
$$

Since $\Psi$ is monotonically nondecreasing and con-

cave, then, by (40),

$$
\begin{gathered}
\Psi\left[\frac{1}{B_{K}+C_{K}} \sum_{\alpha \in K}\left(b_{\alpha}+c_{\alpha}\right) f\left(w_{\alpha}\right)-f\left(\frac{1}{\left(1 / B_{K}+B_{K}\right) \sum_{\alpha \in K}\left(b_{\alpha}+c_{\alpha}\right) / w_{\alpha}}\right)\right] \\
\geq \frac{B_{K} \Psi\left[\left(1 / B_{K}\right) \sum_{\alpha \in K} b_{\alpha} f\left(w_{\alpha}\right)-f\left(1 /\left(1 / B_{K}\right) \sum_{\alpha \in K} b_{\alpha} / w_{\alpha}\right)\right]}{B_{K}+C_{K}} \\
+\frac{C_{K} \Psi\left[\left(1 / C_{K}\right) \sum_{\alpha \in K} c_{\alpha} f\left(w_{\alpha}\right)-f\left(1 /\left(1 / C_{K}\right) \sum_{\alpha \in K} c_{\alpha} / w_{\alpha}\right)\right]}{B_{K}+C_{K}}
\end{gathered}
$$

which, by multiplication with $B_{K}+C_{K}>0$, produces the desired result (39) for $i=1$.

(ii) If $\mathbf{b} \geq \mathbf{c}$, then by (i),

Similarly, we can easily verify result (39), for $i=2$.

$$
\begin{aligned}
\mathscr{D}_{i}(f, K, \mathbf{b}, \mathbf{w} ; \Psi) & =\mathscr{D}_{i}(f, K,(\mathbf{b}-\mathbf{c})+\mathbf{c}, \mathbf{w} ; \Psi) \\
& \geq \mathscr{D}_{i}(f, K,(\mathbf{b}-\mathbf{c}), \mathbf{w} ; \Psi)+\mathscr{D}_{i}(f, K, \mathbf{c}, \mathbf{w} ; \Psi) \geq \mathscr{D}_{i}(f, K, \mathbf{c}, \mathbf{w} ; \Psi),
\end{aligned}
$$

since $\mathscr{D}_{i}(f, K,(\mathbf{b}-\mathbf{c}), \mathbf{w} ; \Psi) \geq 0$.

Corollary 2. Let $f \in H \operatorname{Conv}(C, \mathbb{R}), K \in P_{f}(\mathbb{N}), \mathbf{w} \in J_{*}(C)$, and $\Psi:(0, \infty) \longrightarrow \mathbb{R}$ be a monotonically nondecreasing and concave function.
If there exist the numbers $m$ and $M$ with $M \geq m \geq 0$ such that $M \mathbf{c} \geq \mathbf{b} \geq m \mathbf{c}$, then

$$
\begin{aligned}
& M C_{K} \Psi\left[\frac{1}{C_{K}} \sum_{\alpha \in K} c_{\alpha} f\left(w_{\alpha}\right)-f\left(\frac{1}{\left(1 / C_{K}\right) \sum_{\alpha \in K} c_{\alpha} / w_{\alpha}}\right)\right] \\
& \geq B_{K} \Psi\left[\frac{1}{B_{K}} \sum_{\alpha \in K} b_{\alpha} f\left(w_{\alpha}\right)-f\left(\frac{1}{\left(1 / B_{K}\right) \sum_{\alpha \in K} b_{\alpha} / w_{\alpha}}\right)\right] \\
& \geq m C_{K} \Psi\left[\frac{1}{C_{K}} \sum_{\alpha \in K} c_{\alpha} f\left(w_{\alpha}\right)-f\left(\frac{1}{\left(1 / C_{K}\right) \sum_{\alpha \in K} c_{\alpha} / w_{\alpha}}\right)\right] .
\end{aligned}
$$

In particular, 


$$
\begin{aligned}
& \frac{M}{m} \Psi\left[\frac{1}{C_{K}} \sum_{\alpha \in K} c_{\alpha} f\left(w_{\alpha}\right)-f\left(\frac{1}{\left(1 / C_{K}\right) \sum_{\alpha \in K} c_{\alpha} / w_{\alpha}}\right)\right] \\
& \geq \Psi\left[\frac{1}{B_{K}} \sum_{\alpha \in K} b_{\alpha} f\left(w_{\alpha}\right)-f\left(\frac{1}{\left(1 / B_{K}\right) \sum_{\alpha \in K} b_{\alpha} / w_{\alpha}}\right)\right] \\
& \geq \frac{m}{M} \Psi\left[\frac{1}{C_{K}} \sum_{\alpha \in K} c_{\alpha} f\left(w_{\alpha}\right)-f\left(\frac{1}{\left(1 / C_{K}\right) \sum_{\alpha \in K} c_{\alpha} / w_{\alpha}}\right)\right] .
\end{aligned}
$$

For a function $\Phi:(0, \infty) \longrightarrow(0, \infty)$, we now consider the functionals

$$
\begin{aligned}
\mathfrak{D}_{1}(f, K, \mathbf{b}, \mathbf{w} ; \Psi, \Phi):= & \sum_{\alpha \in K} \Phi\left(b_{\alpha}\right) \Psi\left[\frac{1}{\sum_{\alpha \in K} \Phi\left(b_{\alpha}\right)} \sum_{\alpha \in K} \Phi\left(b_{\alpha}\right) f\left(w_{\alpha}\right)-f\left(\frac{1}{\left(1 / \sum_{\alpha \in K} \Phi\left(b_{\alpha}\right)\right) \sum_{\alpha \in K} \Phi\left(b_{\alpha}\right) / w_{\alpha}}\right)\right] \\
\mathfrak{D}_{2}(f, K, \mathbf{b}, \mathbf{w} ; \Psi, \Phi):= & \sum_{\alpha \in K} \Phi\left(b_{\alpha}\right) \Psi\left[f\left(w_{1}\right)+f\left(w_{n}\right)-\left(1 / \sum_{\alpha \in K} \Phi\left(b_{\alpha}\right)\right) \sum_{\alpha \in K} \Phi\left(b_{\alpha}\right) f\left(w_{\alpha}\right)\right. \\
& \left.-f\left(\frac{1}{\left(1 / w_{1}\right)+\left(1 / w_{n}\right)-\left(1 / \sum_{\alpha \in K} \Phi\left(b_{\alpha}\right)\right) \sum_{\alpha \in K} \Phi\left(b_{\alpha}\right) / w_{\alpha}}\right)\right]
\end{aligned}
$$

where $f \in \operatorname{HConv}(C, \mathbb{R}), \quad K \in P_{f}(\mathbb{N}), \quad w \in J_{*}(C)$, and $\mathbf{b} \in J^{+}(\mathbb{R})$. Now, if we denote by $\Phi(\mathbf{b})$ the sequence $\left\{\Phi\left(b_{\alpha}\right)\right\}_{k \in \mathbb{N}}$, then we observe that, for $i=1,2$,

$$
\mathfrak{D}_{i}(f, K, \mathbf{b}, \mathbf{w} ; \Psi, \Phi)=\mathscr{D}_{i}(f, K, \Phi(\mathbf{b}), \mathbf{w} ; \Psi) .
$$

The following result may be stated.

Corollary 3. Let $f \in H \operatorname{Conv}(C, \mathbb{R}), K \in P_{f}(\mathbb{N}), \mathbf{w} \in J_{*}(C)$, and $\Psi:(0, \infty) \longrightarrow(0, \infty)$ be monotonically nondecreasing

$$
\begin{aligned}
\mathfrak{D}_{i}(f, K, t \mathbf{b}+(1-t) \mathbf{c}, \mathbf{w} ; \Psi, \Phi) & =\mathscr{D}_{i}(f, K, \Phi(t \mathbf{b}+(1-t) \mathbf{c}), \mathbf{w} ; \Psi) \\
& \geq \mathscr{D}_{i}(f, K, t \Phi(\mathbf{b})+(1-t) \Phi(\mathbf{c}), \mathbf{w} ; \Psi) \\
& \geq \mathscr{D}_{i}(f, K, t \Phi(\mathbf{b}), \mathbf{w} ; \Psi)+\mathscr{D}_{i}(f, K,(1-t) \Phi(\mathbf{c}), \mathbf{w} ; \Psi) \\
& =t \mathscr{D}_{i}(f, K, \Phi(\mathbf{b}), \mathbf{w} ; \Psi)+(1-t) \mathscr{D}_{i}(f, K, \Phi(\mathbf{c}), \mathbf{w} ; \Psi) \\
& =t \mathfrak{D}_{i}(f, K, \mathbf{b}, \mathbf{w} ; \Psi, \Phi)+(1-t) \mathfrak{D}_{i}(f, K, \mathbf{c}, \mathbf{w} ; \Psi, \Phi),
\end{aligned}
$$

for any $\mathbf{b}, \mathbf{c} \in J^{+}(\mathbb{R})$ and $t \in[0,1]$, which proves the statement.

The following result concerning the superadditivity and monotonicity of the functional $\mathscr{D}_{i}(f, K, \mathbf{b}, \mathbf{w} ; \Psi)$, for $i=1,2$, as an index set function holds.
Theorem 9. Let $f \in H \operatorname{Conv}(C, \mathbb{R}), K \in P_{f}(\mathbb{N}), \mathbf{w} \in J_{*}(C)$, and $\mathbf{b} \in J^{+}(\mathbb{R})$. Assume that $\Psi:(0, \infty) \longrightarrow(0, \infty)$ is a monotonically nondecreasing and concave function.

(i) If $K, L \in P_{f}(\mathbb{N})$ with $K \cap L \neq \phi$,

$$
\mathscr{D}_{i}(f, K \cup L, \mathbf{b}, \mathbf{w} ; \Psi) \geq \mathscr{D}_{i}(f, K, \mathbf{b}, \mathbf{w} ; \Psi)+\mathscr{D}_{i}(f, L, \mathbf{b}, \mathbf{w} ; \Psi)
$$

That is, $\mathscr{D}_{i}(f, K, ., \mathbf{w} ; \Psi)$ is superadditive as an index set function on $P_{f}(\mathbb{N})$. (ii) If $K, L \in P_{f}(\mathbb{N})$ with $L \subset K$ and $\Psi:(0, \infty) \longrightarrow$ $(0, \infty)$, then 


$$
\mathscr{D}_{i}(f, K, \mathbf{b}, \mathbf{w} ; \Psi) \geq \mathscr{D}_{i}(f, L, \mathbf{b}, \mathbf{w} ; \Psi)(\geq 0) .
$$

That is, $\mathscr{D}_{i}(f, K, ., \mathbf{w} ; \Psi)$ is monotonically nondecreasing as an index set function on $P_{f}(\mathbb{N})$.
Proof

(i) Let $K, L \in P_{f}(\mathbb{N})$ with $K \cap L \neq \phi$. By the harmonic convexity of $f$ on $C$, we have

$$
\begin{aligned}
\frac{1}{P_{K \cup L}} \sum_{j \in K \cup L} b_{\alpha} f\left(w_{\alpha}\right)-f\left(\frac{1}{\left(1 / P_{K \cup L}\right) \sum_{j \in K \cup L} b_{\alpha} / w_{\alpha}}\right) \\
=\frac{B_{K}\left(\left(1 / B_{K}\right) \sum_{\alpha \in K} b_{\alpha} f\left(w_{\alpha}\right)\right)+P_{L}\left(\left(1 / P_{L}\right) \sum_{l \in L} q_{l} f\left(x_{l}\right)\right)}{B_{K}+P_{L}} \\
\quad-f\left(\frac{1}{\left(B_{K} / B_{K}+P_{L}\right) \cdot\left(1 / B_{K}\right) \sum_{\alpha \in K} b_{\alpha} / w_{\alpha}+\left(P_{L} / B_{K}+P_{L}\right) \cdot\left(1 / P_{L}\right) \sum_{l \in L} q_{l} / x_{l}}\right) \\
\geq \frac{B_{K}\left(\left(1 / B_{K}\right) \sum_{\alpha \in K} b_{\alpha} f\left(w_{\alpha}\right)\right)+P_{L}\left(\left(1 / P_{L}\right) \sum_{l \in L} q_{l} f\left(x_{l}\right)\right)}{B_{K}+P_{L}} \\
\quad-\frac{B_{K} f\left(1 /\left(1 / B_{K}\right) \sum_{\alpha \in K} b_{\alpha} / w_{\alpha}\right)+P_{L} f\left(1 /\left(1 / P_{L}\right) \sum_{l \in L} q_{l} / x_{l}\right)}{B_{K}+P_{L}} \\
=\frac{B_{K}\left[\left(1 / B_{K}\right) \sum_{\alpha \in K} b_{\alpha} f\left(w_{\alpha}\right)-f\left(1 /\left(1 / B_{K}\right) \sum_{\alpha \in K} b_{\alpha} / w_{\alpha}\right)\right]}{B_{K}+P_{L}} \\
\quad+\frac{P_{L}\left[\left(1 / P_{L}\right) \sum_{l \in L} q_{l} f\left(x_{l}\right)-f\left(1 /\left(1 / P_{L}\right) \sum_{l \in L} q_{l} / x_{l}\right)\right]}{B_{K}+P_{L}} .
\end{aligned}
$$

Since $\Psi$ is monotonically nondecreasing and concave, then, by (50),

$$
\begin{aligned}
\Psi\left[\frac{1}{P_{K \cup L}} \sum_{j \in K \cup L} b_{\alpha} f\left(w_{\alpha}\right)-f\left(\frac{1}{\left(1 / P_{K \cup L}\right) \sum_{j \in K \cup L} b_{\alpha} / w_{\alpha}}\right)\right] \\
\geq \frac{B_{K} \Psi\left[\left(1 / B_{K}\right) \sum_{\alpha \in K} b_{\alpha} f\left(w_{\alpha}\right)-f\left(1 /\left(1 / B_{K}\right) \sum_{\alpha \in K} b_{\alpha} / w_{\alpha}\right)\right]}{B_{K}+P_{L}} \\
+\frac{P_{L} \Psi\left[\left(1 / P_{L}\right) \sum_{l \in L} q_{l} f\left(x_{l}\right)-f\left(1 /\left(1 / P_{L}\right) \sum_{l \in L} q_{l} f\left(x_{l}\right)\right)\right]}{B_{K}+P_{L}},
\end{aligned}
$$

which, by multiplication with $B_{K}+P_{L}>0$, produces the desired result (48) for $i=1$.

(ii) Let $K, L \in P_{f}(\mathbb{N})$ with $L \subset K$; then,

Similarly, we can easily verify result (48), for $i=2$.

$$
\begin{aligned}
\mathscr{D}_{i}(f, K, \mathbf{b}, \mathbf{w} ; \Psi) & =\mathscr{D}_{i}(f,(K \backslash L) \cup L, \mathbf{b}, \mathbf{w} ; \Psi) \geq \mathscr{D}_{i}(f, K \backslash L, \mathbf{b}, \mathbf{w} ; \Psi)+\mathscr{D}_{i}(f, L, \mathbf{b}, \mathbf{w} ; \Psi) \\
& \geq \mathscr{D}_{i}(f, L, \mathbf{b}, \mathbf{w} ; \Psi)(\geq 0),
\end{aligned}
$$

since $\mathscr{D}_{i}(f, K \backslash L, \mathbf{b}, \mathbf{w} ; \Psi) \geq 0$. 


\section{Conclusion}

First of all, we have presented the refinement of Jensen-type inequality, and further, we have discussed several important aspect of functionals associated with Jensen-type inequalities for the HCFs. On the basis of ideas discussed in this paper along with the literature present on HCFs, we encourage the interested researcher to explore more interesting results for this class of functions.

\section{Data Availability}

No data were used to support this study.

\section{Conflicts of Interest}

The authors declare that there are no conflicts of interest regarding the publication of this paper.

\section{Authors' Contributions}

All authors contributed equally in this research article. All authors read and approved the final manuscript.

\section{References}

[1] İ. İşcan, "Hermite-Hadamard type inequaities for HCFs," Hacettepe Journal of Mathematics and Statistic, vol. 43, no. 6, pp. 935-942, 2014.

[2] İ. İşcan, "Hermite-Hamard type inequalities for harmonically $(\alpha, m)$-convex functions," 2015, http://arxiv.org/abs/1307. $5402 \mathrm{v} 3$.

[3] I. A. Baloch, I. Işcan, and S. S. Dragomir, "Fejer's type inequalities for harmonically $(s, m)$-convex functions," International Journal of Analysis and Applications, vol. 12, pp. 188-197, 2016.

[4] I. A. Baloch and I. Işcan, "Some ostrowski type inequalities for harmonically $(s, m)$-convex functions in second sense," International Journal of Analysis, vol. 2015, Article ID 672675, 9 pages, 2015 .

[5] I. A. Baloch and I. Işcan, "Some Hermite-Hadamard type integral inequalities for Harmonically $(p,(s, m))$-convex functions," Journal of Inequalities and Special Functions, vol. 8, pp. 65-84, 2017.

[6] S. H. Wu, I. A. Baloch, and I. Iscan, "On harmonically $(p, h, m)$-preinvex functions," Journal of Function Spaces, vol. 2017, no. 3, Article ID 2148529, 2017.

[7] I. A. Baloch, M. De La Sen, and İ. İşcan, "Characterizations of classes of HCFs and applications," International Journal of Analysis and Applications, vol. 17, pp. 722-733, 2019.

[8] N. Akhtar, M. U. Awan, M. Z. Javed et al., "Ostrowski type inequalities involving harmonically convex functions and applications," Symmetry, vol. 13, 2021.

[9] J. Z. Xu, U. Raza, M. W. Javed, and Z. Hussain, "HermiteHadamard inequalities for harmonic $(s, m)$-convex functions," Mathematical Problems in Engineering, vol. 2020, Article ID 1470837, 7 pages, 2020.

[10] S. I. Butt, S. Rashid, M. Tariq, and M. K. Wang, "Novel refinements via $n$-polynomial harmonically $s$ type convex functions and application in special functions," Journal of Function Spaces, vol. 2021, Article ID 6615948, 17 pages, 2021.

[11] E. R. Nwaeze, M. A. Khan, M. Ahmadian, M. Nazir Ahmad, and A. Kamil Mahmood, "Fractional inequalities of the
Hermite-Hadamard type for \$ $\mathrm{m}$ \$-polynomial convex and harmonically convex functions," AIMS Mathematics, vol. 6, no. 2, pp. 1889-1904, 2021.

[12] S. I. Butt, M. Tariq, A. Aslam, H. Ahmad, and T. A. Nofal, "Hermite Hadamard type inequalities via generalized harmonic exponential convexity and applications," Journal of Function Spaces, vol. 2021, Article ID 5533491, 12 pages, 2021.

[13] Y. M. Chu, S. Rashid, T. Abdeljawad, A. khalid, and H. Kalsoom, "On new generalized unified bounds via generalized exponentially harmonically $s$-convex functions on fractal sets," Advances in Difference Equations, vol. 218, 2021.

[14] I. A. Baloch, A. A. Mughal, Y. M. Chu, A. U. Haq, and M. D. L. Sen, "Improvement and generalization of some results related to the class of harmonically convex functions and applications," Journal of Mathematics and Computer Science, vol. 22, no. 3, pp. 282-294, 2021.

[15] S. Varošanec, "On h-convexity," Journal of Mathematical Analysis and Applications, vol. 326, no. 1, pp. 303-311, 2007.

[16] Z. B. Fang and R. Shi, "On the $(p, h)$-convex function and some integral inequalities," Journal of Inequalities and Applications, vol. 2014, no. 1, 45 pages, 2014.

[17] A. Hśzy, "Berstein-doetsch type results for $h$-convex functions," Mathematical Inequalities \& Applications, vol. 14, no. 3, pp. 499-508, 2011.

[18] A. Olberyś, "On separation by $h$-convex functions," Tatra Mountains Mathematical Publications, vol. 62, no. 1, pp. 105-111, 2015.

[19] M. U. Awan, M. A. Noor, M. V. Mihai, K. I. Noor, and A. G. Khan, "Some new bounds for Simpson's rule involving special functions via harmonic $h$-convexity," The Journal of Nonlinear Sciences and Applications, vol. 10, no. 4, pp. 1755-1766, 2017.

[20] M. R. Delavar, S. S. Dragomir, and M. De La Sen, "A note on characterization of $h$-convex functions via hermite-hadamard type inequality," Issues of Analysis, vol. 26, no. 2, pp. 28-36, 2019.

[21] S. S. Dragomir, "Inequalities of Jensen type for $H A$-convex functions," Analele Universitati Oradea Fasc. Matematica, vol. 27, pp. 103-124, 2020.

[22] I. Abbas Baloch, A. H. Mughal, A. Ahmad Mughal, Y.-M. Ul Haq, and M. De La Sen, "A variant of Jensen-type inequality and related results for harmonic convex functions," Aims Mathematics, vol. 5, no. 6, pp. 6404-6418, 2020.

[23] R. P. Agarwal and S. S. Dragomir, "The property of supermultiplicity for some classical inequalities and applications," Computers \& Mathematics with Applications, vol. 35, no. 6, 105118 pages, 1998.

[24] S. S. Dragomir, J. E. Pećarić, and L. E. Persson, "Some inequalities of Hadamard inequality," Soochow Journal of Mathematics, vol. 21, no. 3, pp. 335-341, 1995.

[25] S. S. Dragomir, "Bounds for the normalized Jensen functional," Bulletin of the Australian Mathematical Society, vol. 74, no. 3, pp. 471-478, 2006.

[26] S. S. Dragomir, J. Pećarić, and L. E. Persson, "Properties of some functionals related to Jensens inequality," Acta Mathematica Hungarica, vol. 70, no. 12, pp. 129-143, 1996.

[27] P. Agarwal, M. Kadakal, İ. İşcan, and Y. M. Chu, "Better approaches for $n$-times differentiable convex functions," Mathematics, vol. 8, 2020.

[28] M. Tomar, P. Agarwal, and J. Choi, "Hermite-Hadamard type inequalities for generalized convex functions on fractal sets style," Boletim da Sociedade Paranaense de Matemtica, vol. 38, pp. 101-116, 2020. 
[29] M. B. Khan, M. A. Noor, K. I. Noor, H. Almusawa, and K. S. Nisar, "Exponentially preinvex fuzzy mappings and fuzzy exponentially mixed variational-like inequalities," International Journal of Analysis and Applications, vol. 19, pp. 518541, 2021.

[30] H. Almusawa, H. A. Hammad, and N. Sharma, "Approximation of the fixed point for unified three-step iterative algorithm with convergence analysis in busemann spaces," Axioms, vol. 10, no. 1, 26 pages, 2021. 